\title{
Imaging the coupling of terahertz radiation to a high electron mobility transistor in the near-field
}

M. Ortolani

michele.ortolani@ifn.cnr.it

\section{A. Di Gaspare \\ E. Giovine \\ F. Evangelisti}

\section{Foglietti}

\section{A. Doria \\ G. P. Gallerano \\ E. Giovenale \\ G. Messina}

\section{Spassovsky}

C. Lanzieri

\section{Peroni}

\section{A. Cetronio}

CNR - Istituto di Fotonica e Nanotecnologie, Via Cineto Romano 42, 00156 Rome, Italy

CNR - Istituto di Fotonica e Nanotecnologie, Via Cineto Romano 42, 00156 Rome, Italy

CNR - Istituto di Fotonica e Nanotecnologie, Via Cineto Romano 42, 00156 Rome, Italy

CNR - Istituto di Fotonica e Nanotecnologie, Via Cineto Romano 42, 00156 Rome, Italy

CNR - Istituto di Fotonica e Nanotecnologie, Via Cineto Romano 42, 00156 Rome, Italy

ENEA - Centro Ricerche Frascati, 00044 Frascati, Italy

ENEA - Centro Ricerche Frascati, 00044 Frascati, Italy

ENEA - Centro Ricerche Frascati, 00044 Frascati, Italy

ENEA - Centro Ricerche Frascati, 00044 Frascati, Italy

ENEA - Centro Ricerche Frascati, 00044 Frascati, Italy

Selex Sistemi Integrati, Via Tiburtina Km 12.400, 00131 Rome, Italy

Selex Sistemi Integrati, Via Tiburtina Km 12.400, 00131 Rome, Italy

Selex Sistemi Integrati, Via Tiburtina Km 12.400, 00131 Rome, Italy

We used AlGaN/GaN high electron mobility transistors as room-temperature direct detectors of radiation at $0.15 \mathrm{THz}$ from a free electron laser, hence 5 times higher than their cutoff frequency of $30 \mathrm{GHz}$. By near-field active mapping we investigated the antenna-like coupling of the radiation to the transistor channel. We formulate a model for the detection based on self-mixing in the transistor channel. The noise equivalent power is found in the range of $10^{-7} \mathrm{~W} / \mathrm{Hz}^{0.5}$ without any optimization of the device responsivity. Present day AlGaN/GaN fabrication technology may provide operation at higher frequency, integration of amplifiers for improved responsivity and fast switches for multiplexing, which make the detector here described the basic element of a monolithic terahertz focal plane array. [DOI: 10.2971/jeos.2009.09006]

Keywords: terahertz, heterostructure, detector, near-field

\section{INTRODUCTION}

The rapid development of compact terahertz sources and detectors suggests a large number of practical imaging applications for non-destructive material testing, medical diagnostics and security. Nowadays, commercial instruments start to appear in the millimeter-wave range $(0.1-0.3 \mathrm{THz})$. The acquisiton of images with a large field of view relies either on raster-scanning with highly sensitive, low-temperature singlepixel detectors, or on massive arrays of modular detectors, based on passive rectifying elements like Schottky diodes or semiconductor-metal-semiconductor junctions. Therefore, a room-temperature integrated focal plane array (FPA) detector is desirable to achieve a compact video-rate terahertz im- ager. The most direct way of obtaining an integrated FPA is to look for devices sensitive to terahertz radiation which can be fabricated directly on a semiconductor wafer by fabrication steps compatible with present-day high-speed semiconductor technology [1]. In the last years, high electron mobility transistors (HEMTs) have been demonstrated to operate as detectors of terahertz radiation well beyond their cutoff frequency for amplification $f_{T}$ [2], displaying gate-tunable resonant detection [3] and detecting radiation up to $3.1 \mathrm{THz}$ when cooled at low temperatures [4]. The detection mechanism is based on the properties of the two-dimensional electron gas found at the interface of the semiconductor heterostructure on 
which the HEMT is fabricated, which provides operation as either direct detector [2] or as mixer [5]. From the noise and responsivity figures reported [1], at room temperature a quartermicron gate AlGaAs/GaAs HEMT should be sensitive to a flux of tens of $\mu \mathrm{W} / \mathrm{mm}^{2}$ in the millimeter-wave range, which can be obtained from diode-based frequency multipliers. It seems therefore promising to design an integrated FPA to be fabricated on a semiconductor heterostructure wafer like AlGaAs/GaAs or AlGaN/GaN, together with low-noise amplifiers and fast switches for readout which are already achievable on these materials. Extension of the FPA operation to higher frequency and/or lower flux are not precluded in principle, and may require only minor changes to the fabrication process.

The first step towards an integrated FPA is the study of the detection of terahertz radiation by single HEMTs in order to optimize the radiation coupling and to fit the data to a phenomenological model, which allows then for further design and simulation. In this paper, we demonstrate the operation of a AlGaN/GaN HEMT at room temperature as a direct detector of radiation at $0.15 \mathrm{THz}$, i. e. at a frequency higher than its $f_{T}=30 \mathrm{GHz}$. The radiation coupling to the transistor channel was investigated by active near-field imaging with a lateral resolution of $200 \mu \mathrm{m}$ ( $\lambda / 10$ where $\lambda$ is the wavelength). The detection mechanism is attributed to self-mixing effect of the signal provided by a single source (in this case a Free Electron Laser (FEL)), simultaneously coupled to the transistor channel and to the gate electrode. As a result, direct dc detection of the source power is obtained. The operation frequency of $0.15 \mathrm{THz}$ was set by the source itself, and operation at higher frequency may be possible with the same HEMT or by implementing shorter gate length and lithographic antennas in an advanced design.

\section{EXPERIMENTAL SETUP}

Double-channel HEMTs were fabricated on AlGaN/GaN heterostructures grown on $\mathrm{SiC}$ substrates. A photograph of the device is shown in Figure 1(a) and displays two source pads, one drain pad and one gate pad with two $0.25 \mu \mathrm{m}$ long Schottky gate fingers. The channel width is $50 \mu \mathrm{m}$. The device fabrication technology is based on a mix and match approach by using both stepper and electron beam lithography [6]. The schematic of the circuit used for the double-channel HEMT is shown in Figure 1(b) and includes dc bias power supplies $\left(V_{c}=10 \mathrm{~V}\right.$ and variable $\left.V_{g 0}\right)$. The two source pads (S1, S2), the drain (D) and gate $(G)$ pads were wire-bonded through $1.5 \mathrm{~mm}$ long, $25 \mu \mathrm{m}$ diameter, aluminium wires to the copper lines of a PC board, and these were connected via coaxial cables to the voltage sources. The dc current $i_{d 0}$ flowing through the channel and a series resistor $R_{d}=1.02 \mathrm{k} \Omega$ was measured with a digital meter for all $V_{g 0}$ and the static drain voltage was obtained by $V_{d 0}=V_{c}-i_{d 0} R_{d}$.

The near-field imaging system developed at the ENEA-FEL facility in Frascati [7]-[9] generates radiation at $0.15 \mathrm{THz}$ collected by a series of two WR5 directional couplers terminated by a microprobe open waveguide (WG) end, which directs the radiation to the surface of the sample under investigation. The
WG has a rectangular section of $1.2 \times 0.6 \mathrm{~mm}^{2}$. The FEL pulse duration is $3 \mu \mathrm{sec}$ and the repetition rate is $2 \mathrm{~Hz}$. Triggered data acquisition takes place at every single FEL shot. A radiation monitor (fast Schottky diode) is inserted inside the WG and used to measure the FEL power and pulse shape at each shot. The HEMT chip was positioned just below the open WG end and exposed to the FEL radiation pulses. The transient drain signal $V_{d}(t)$ was then acquired by a digital oscilloscope.
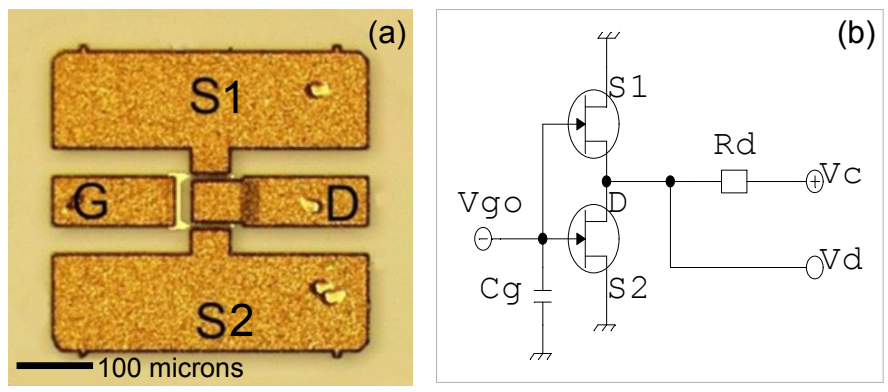

FIC. 1 (a) Photograph of the double channel HEMT trough an optical microscope with metal Source (S) and Drain (D) pads and gate fingers elongated from pad $G$ over the two channel areas. (b) Circuit for the $150-\mathrm{GHz}$ detection experiment.

In Figure 2 the transient drain voltage $V_{d}(t)-V_{d 0}$ during a single FEL pulse illumination is shown for several values of $V_{g 0}$. For $V_{g 0}=-4.5 \mathrm{~V}$ and $i_{d 0}=4.91 \mathrm{~mA}$ the maximum absolute value of the signal is obtained, i. e. close to the transistor pinch-off at $V_{g 0} \leq-5.6 \mathrm{~V}$. The signals in $V_{d}(t)$ are simultaneous to the FEL pulse, as they temporally overlap with the monitor signal from the diode installed inside the WG. The absolute variation of $V_{d}(t)$ around $1 \mathrm{~V}$ is comparable to the static values $V_{g 0}$ and $V_{d 0}$. This fact indicates that the transistor parameters, e. $g$. the transconductance $g_{m}=\partial i_{d} / \partial V_{g}$, are being modulated by the strong electric field of the radiation.

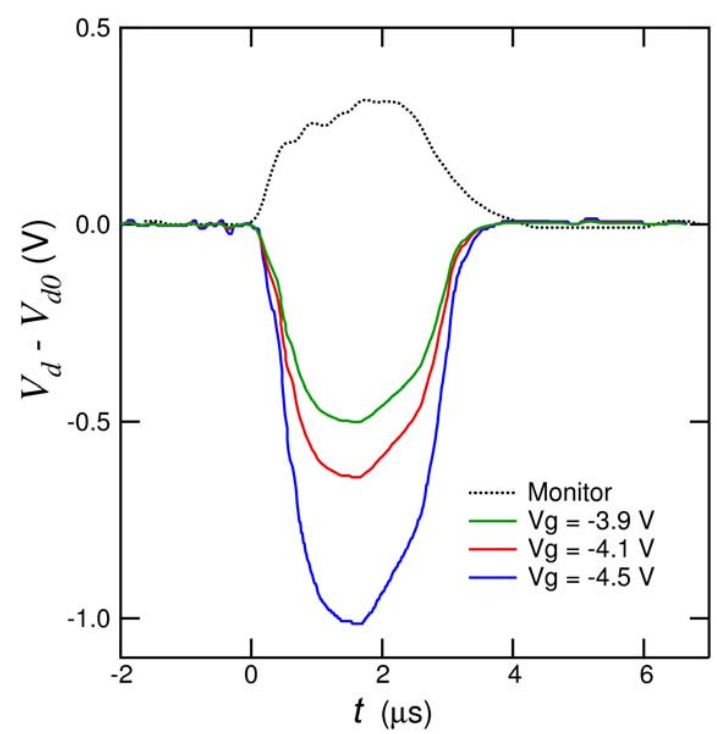

FIG. 2 Typical transient signals from the HEMT in the saturation region (negative values). The dotted line is the simultaneous signal from the radiation monitor installed inside the waveguide. 


\section{ACTIVE IMAGING OF RADIATION COUPLING}

The normal operation of the ENEA-FEL instrument for nondestructive material testing is the imaging of the power reflected by a sample, which is raster-scanned below the WG end by keeping constant the WG-to-sample distance $z$ [8]. For each position, one FEL shot is fired and the emitted power is measured by the monitor diode. The $z$-dependent percentage of reflected power $R_{z}$ is measured at every given position by a second Schottky diode mounted close to the WG end (passive imaging) and a $R_{z}$-map is obtained. If $z<\lambda$, the sample is in the near-field region and a lateral resolution down to $200 \mu \mathrm{m}$, i. e. better than $\lambda$, can be achieved. In the present experiment, we imaged the radiation coupling to the HEMT detector by recording the signal measured by the detector itself at every given position, while raster-scanning the detector board below the open WG-end (active imaging [9]). Here also, since the distance between the HEMT and the open WG-end is much smaller than $\lambda$, we are imaging the near-field coupling of the radiation to the detector at a lateral resolution better than $\lambda$ [9]-[11]. The transient drain voltage during the FEL pulse is recorded by a digital oscilloscope as in Figure 2 and the detector signal $V^{*}$ is calculated by

$$
V^{*}=\int_{0}^{\Delta t}\left(V_{d}(t)-V_{d 0}\right) d t
$$

where $t=0$ is given by the FEL trigger and $\Delta t=3 \mu \mathrm{s}$. The passive $\left(R_{z}\right)$ image of the detector was also obtained, an example of which is shown in Figure 3(a). The WG-to-board distance $z$ was set to $1.0 \mathrm{~mm}(\lambda / 2)$ so that the reflected power is maximum when the WG is above the copper lines on the PC board, while almost no power is reflected by the chip. The position of the copper lines was used to assign coordinates to the HEMT chip and the bonding wires. In this way, we could superimpose the active $\left(V^{*}\right)$ images obtained for different bias and polarization conditions to a map describing the position of the chip and the wires. The result of this procedure is shown in Figure 3(b).

In a first experiment, we connected the HEMT as depicted in Figure 1. The active map in Figure 3(b) displays a broad spot centered above the HEMT. A straightforward conclusion would be that since the HEMT is acting as a detector, the maximum signal is obtained when the WG is on top of it. However, a closer inspection of Figure 3(b) indicates that the area of maximum signal is significantly larger than both the HEMT active area $\left(0.3 \times 0.3 \mathrm{~mm}^{2}\right.$ including contact pads $)$ and the WG end $\left(1.2 \times 0.6 \mathrm{~mm}^{2}\right)$, suggesting that the radiation is also coupled to the transistor channel through the bonding wires (red lines in Figure 3), by a high-frequency electromagnetic pick-up mechanism which makes the wires to act as antennas. Recently, a similar effect has been indirectly observed when exposing a AlGaAs/GaAs HEMT to a linearly polarized beam at $100 \mathrm{GHz}$ and compared to finite element calculations [12]. According to the authors, the effect of bonding wires can be understood in terms of dynamic resistors and capacitors connected between themselves and to the transistor channel, whose values and connections strongly depends on details of the bonding wire geometry. Another equivalent interpretation could be given in terms of unconventional dipole antennas. Indeed, our set-up is the high-frequency analogue of other experimental setups extensively used to extract antenna parameters $[13,14]$ through measurements of mutual coupling between two antennas in the near-field region. It is beyond the scope of this work to investigate the theory and to develop the techniques to calculate the mutual coupling in our experiment, however we emphasize that our set-up can be used to extrapolate data of millimeter-wave antennas and push the limits of the near-field measurements at higher frequencies and lower dimensions with respect to past experiments.

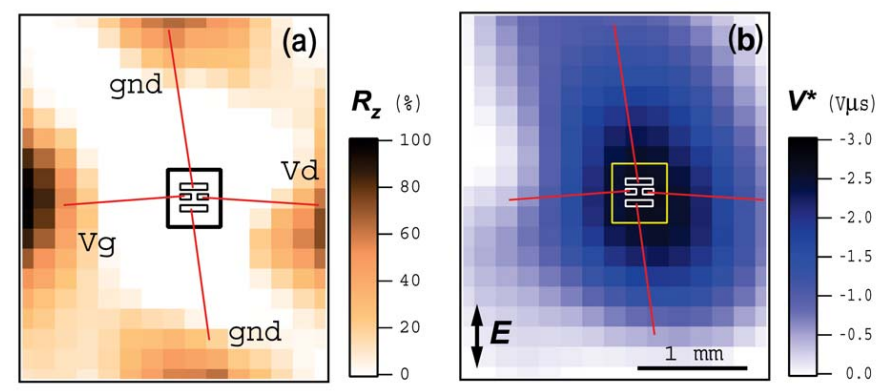

FIG. 3 (a) Passive image of the PC board, where the copper lines are clearly visible. (b) Active near-field image of the integrated HEMT signal $V^{*}$ obtained by scanning the HEMT below the open end waveguide. The HEMT chip, oriented as in Figure 1(a), is sketched as a square. Bonding wires are also sketched with their approximate length and direction. $V_{c}=10 \mathrm{~V}, i_{d 0}=-4.91 \mathrm{~mA}$ and $V_{g 0}=-4.5 \mathrm{~V}$. The arrow in (b) indicates the electric field direction of the FEL radiation.

In a second experiment, we mounted two identical HEMTs on another PC board at a lateral distance equal to $\lambda=2 \mathrm{~mm}$ in order to test cross-talking effects between two "pixels". In that case, only one channel of the HEMT was activated by connecting the D-pad to ground and the S1 pad to the drain circuit, while the S2 pad was left floating. Each HEMT was connected to a different drain circuit (Vd1 and Vd2 in Figure 4) while the ground and the gate circuit were in common. The configuration is shown by the passive image in Figure 4(a). Detection signals similar to those in Figure 2 were obtained by exposing either one or the other HEMT when the corresponding $V_{c}$ was turned on, with a somewhat smaller intensity (the active channel width being half of that of the first experiment). The active images in Figures 4 (b) and 4(c) were obtained with $V_{c}=10$ $\mathrm{V}$ for the top transistor, $V_{c}$ disconnected for the bottom transistor and $V_{g 0}=-4.5 \mathrm{~V}$ applied to both transistors. Interestingly, in Figure 4(b) the maximum signal is found when the WG is outside the HEMT chip, at a position which is just on top of a bonding wire, connecting the drain circuit Vd1 to the S1 pad, and running parallel to the direction of the electric field of the radiation $\mathbf{E}$. After the acquisition of the active image in Figure 4(b), we rotated the PC board with respect to the WG end and recorded another image, shown in Figure 4(c). In this image, the $\mathbf{E}$ direction is effectively rotated by $90^{\circ}$ and one can see that the maximum signal is again obtained on the wire which is parallel to the E direction. To sum up, the active images confirm that the maximum radiation coupling is found on bonding wires running parallel to the E direction, irrespectively of the orientation of the transistor channel. This means that the transistor channel responds more to an elec- 
trical (antenna-like) excitation, rather than to a direct optical excitation. However, the signal $V^{*}$ at the center of the chip is nonzero and it is lower than the maximum just by a factor of ten. Radiation coupling maps might be different at higher frequency, where the electrical pick-up by bonding wires should be negligible [12] and direct detection by the 2-dimensional electron gas in the HEMT is predicted [15].

Finally, it appears in Figure 4(b) that when the bottom transistor, which has $V_{g}=-4.5 \mathrm{~V}$, grounded source and floating $V_{d}$, is exposed to the FEL pulses, a small signal is measured at the drain of the top transistor. This cross-talking effect is probably due to local oscillation of the common gate potential. Another possibility is that, beyond the near-field illumination, the electric field propagates along the PC board far from the openend WG position. This shall be the subject of a subsequent paper. In any case, the data in Figures 4(b) and 4(c) indicate that cross-talking effects are present in the common gate configuration, but they do not represent a major concern.

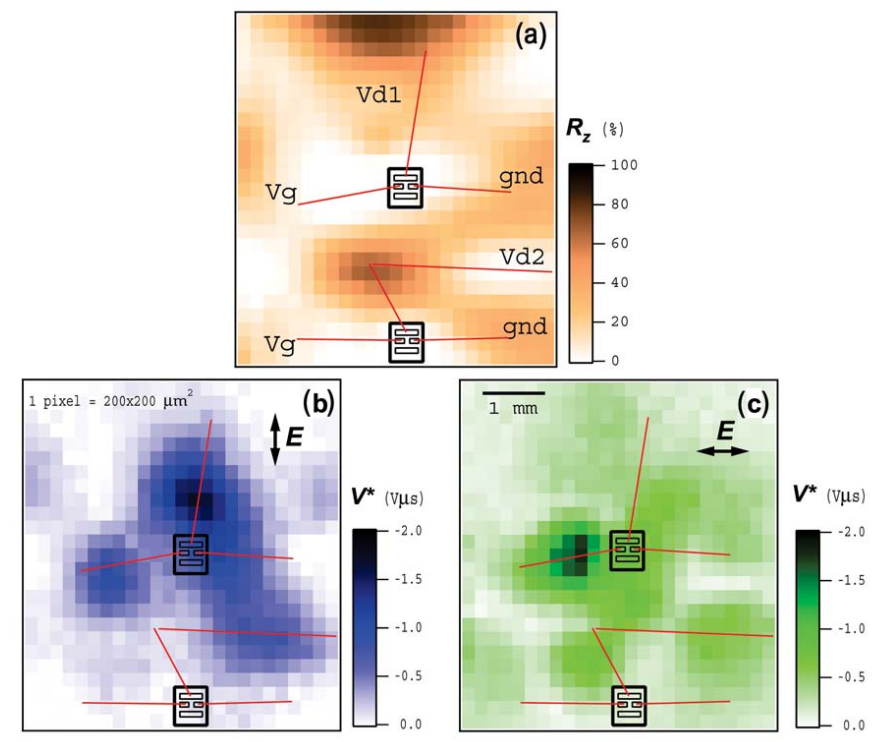

FIG. 4 Passive (a) and active (b,c) near-field images of a two-transistor system. In (c), the FEL radiation electric field direction is effectively rotated by $90^{\circ}$ degrees, as indicated by the arrows. The HEMT chip is sketched as a square and the bonding wires as red lines. Once the symmetry in the transistor circuit is broken, the maximum signal is found when wires parallel to the field are irradiated. $V_{c}=10 \mathrm{~V}$ is applied to the top transistor only, while $V_{g 0}=-4.5 \mathrm{~V}$ is applied to both.

\section{DETECTION MECHANISM}

We now propose a phenomenological model which accounts for the negative signal in $V_{d}(t)$ seen in the saturation region of the transistor characteristics $\left(V_{g 0}=-4.5 \mathrm{~V}, i_{d 0}=4.91 \mathrm{~mA}\right)$ [16]. To sum up the experimental facts: $i$ ) from the data in Figure 2 we have that the maximum signal is found close to the transistor pinch-off; $i$ ) from the images in Figures 3 and 4 we have that the detection signal is due to an electrical signal coupled to the channel by the gate, or drain, or grounded source contact (the latter being less effective, see Figure 4). The detection mechanism in the saturation region relies then on the high frequency $\left(\omega_{h f}=150 \mathrm{GHz}\right)$ component of the drain voltage and current induced by the radiation and whose amplitudes are proportional to the time-varying radiation field $\mathbf{E} e^{\imath \omega_{h f} t}$.
As a consequence of that, a voltage at $\omega_{h f}$ is produced in the channel and this leads to a gate-to-channel voltage variation $v_{g c}(t)=v_{g c} \sin \left(\omega_{h f} t\right.$ ) (in our configuration the gate contact is grounded by an external capacitor $C_{g}$ shown in Figure 1(b) with respect to a time-varying signal, so that the variation $v_{g c}$ is indeed related to the relative variation of the potential between the channel and the gate contact). As a result of the mixing between $v_{g c}(t)$ and the time-varying transistor parameters $[17,18]$, we have a down-converted component of the drain current $i_{d}(t)$ which adds up to the dc bias current $i_{d 0}$ during the FEL pulse illumination. If we are far from the linear region of the transistor characteristics we can consider a series expansion of $g_{m}$ to the first order in $v_{g c}$ and approximate $i_{d}(t)=i_{d 0}+g_{m} \times v_{g c}(t)$ by:

$$
i_{d}(t)-i_{d 0} \simeq g_{m 0} \times v_{g c}(t)+\frac{\partial g_{m}}{\partial v_{g c}} \times v_{g c}^{2}(t)
$$

The first term is the high-frequency linear response, the second one is the square-law self-mixing term displaying one down-converted component at low frequency and one at $2 \omega_{h f}$. After low-pass filtering by parasitic capacitances in our device (whose unity gain is indeed at $30 \mathrm{GHz}$ ), the transient voltage drop $V_{d}(t)$, measured at the drain connector and reported in Figure 2, can be approximated by $V_{d}(t) \simeq$ $-\left\langle i_{d}(t)\right\rangle R_{d}$ (where \langle\rangle indicates the low-pass filtering of the high-frequency components), hence:

$$
V_{d}(t)-V_{d 0} \simeq-\frac{\partial g_{m}}{\partial v_{g c}}\left\langle v_{g c}^{2} \sin ^{2}\left(\omega_{h f} t\right)\right\rangle \times R_{d}
$$

We can now compare the peak signal measured in the first experiment as a function of $V_{g 0}$ with an estimate of the quantity $\partial g_{m} / \partial v_{g c}$. The latter is obtained by double numeric differentation of the $i_{d 0}$ vs. $V_{g 0}$ curve measured in dc (green line in Figure $5(\mathrm{a})$ ). This is a rough approximation, as the transistor characteritics are expected to vary with frequency. Nevertheless, the comparison shown in Figure 5(b) indicates that the main features of the dependence from the gate voltage are captured by our phenomenological model. A region with a positive signal (negative $\partial g_{m} / \partial v_{g c}$ ) is found for $V_{g 0}>-3 \mathrm{~V}$ in both the detection signal (red dots in Figure 5(b)) and the calculated curve (grey line in Figure 5(b)). More importantly, the maximum absolute value of the detection signal is found at $V_{g 0}=$ $-4.5 \mathrm{~V}$ and corresponds to the maximum in $-\partial g_{m} / \partial v_{g c}$. Considering the variation of $g_{m}$ with $V_{g 0}$ in Figure 5(b), the selfmixing effect takes place when approaching the saturation region from small negative $V_{g 0}$ down to the threshold voltage. For low gate bias the E-field may bring the Schottky gate contact forward biased, producing a positive component of $V_{d}(t)$ [9], so that the mixing action can be clearly observed only at large negative $V_{g 0}$.

A further test of the model is the linearity check as a function of the radiation power. To perform this test, we monuted a variable attenuator in the waveguide, before the monitor and the WG end. The pulse energy can be then reduced from its maximum value of $0.25 \mathrm{~mJ}$ over two orders of magnitude in a controlled way for different pulses. By monitoring the detec- 


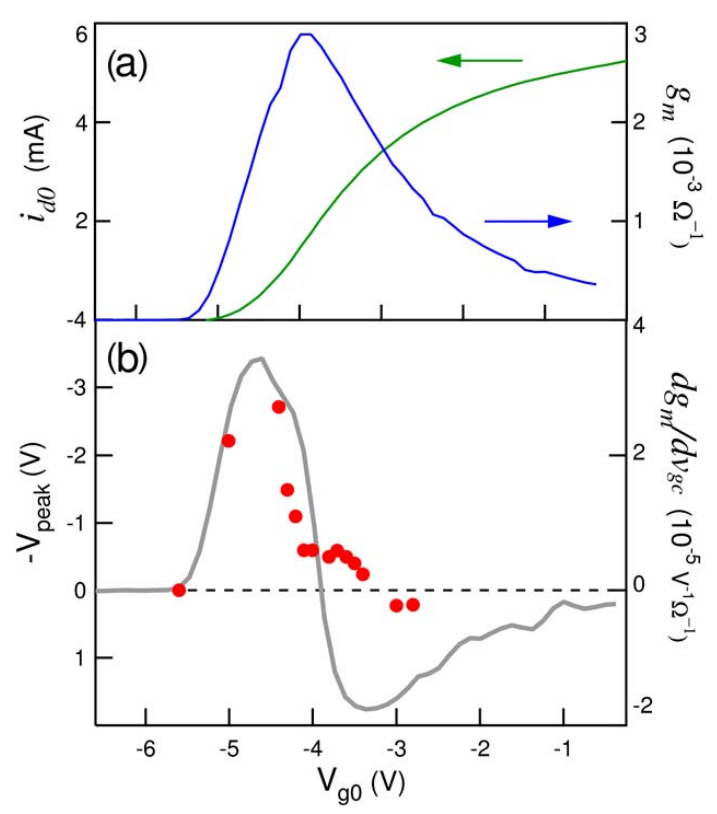

FIG. 5 (a) dc current (green, left scale) and transconductance (blue, right scale) of the HEMT used as a detector, measured without an external load resistor $R_{d}$ and $V_{c}=$ $100 \mathrm{mV}$. (b) Peak detection signal (dots, left scale) compared with the numerically calculated transconductance change (grey line, right scale). The two independently obtained quantities show a maximum in the same range of gate voltage values $V_{g 0}$.

tor response to weaker fields, one can discriminate eventual mechanisms displaying an electric field threshold value, like dielectric breakdown or photoexcitation. In Figure 6 we show the dependence of the peak value in the saturation regime $\left(V_{g 0}=-3.8 \mathrm{~V}\right)$ as a function of the FEL pulse energy, reduced for different pulses by the attenuator. The integrated signal $V^{*}$ scales linearly with the pulse energy over two orders of magnitude (Figure 6(a)). Moreover, the peak output signal scales linearly with the peak power measured by the monitor (Figure 6(b)). The same data can be also displayed as a function of the calculated peak value of the electric field in the waveguide estimated from the peak power. This behaviour rules out the existence of a threshold and is compatible with either bolometric (temperature increase) detection or direct square-law electric field amplitude detection. Indeed, Eq. (3) explains the square-law dependence of $V_{d}(t)$ from $v_{g c}$, which is proportional to the transient E-field, the instantaneous power being proportional to $|\mathbf{E}|^{2}$. However, we have seen (Figures 4(b) and $4(\mathrm{c})$ ) that the maximum signal can be obtained when the wires are irradiated, and the transistor is not. This definitely rules out the bolometric mechanism. One may worry that in the configuration used for the first experiment (data in Figure 3) we have two devices connected in parallel, with the istantaneous radiation field directed in the opposite direction with respect to the flow of $i_{d 0}$ (see Figure 1(b)). The square-law mixing term in Eq. (1) indeed explains while we get a finite $V_{d}(t)$ anyway, since the latter quantity has the same negative polarity for both devices, regardless of the instantaneous sign of the E-field.

Concerning detector performances, the maximum of the detector response is found when the waveguide is within $1 \mathrm{~mm}$ from the transistor position. In this condition, with the PC board at $z=1.0 \mathrm{~mm}$, the transistor surface and the bond-
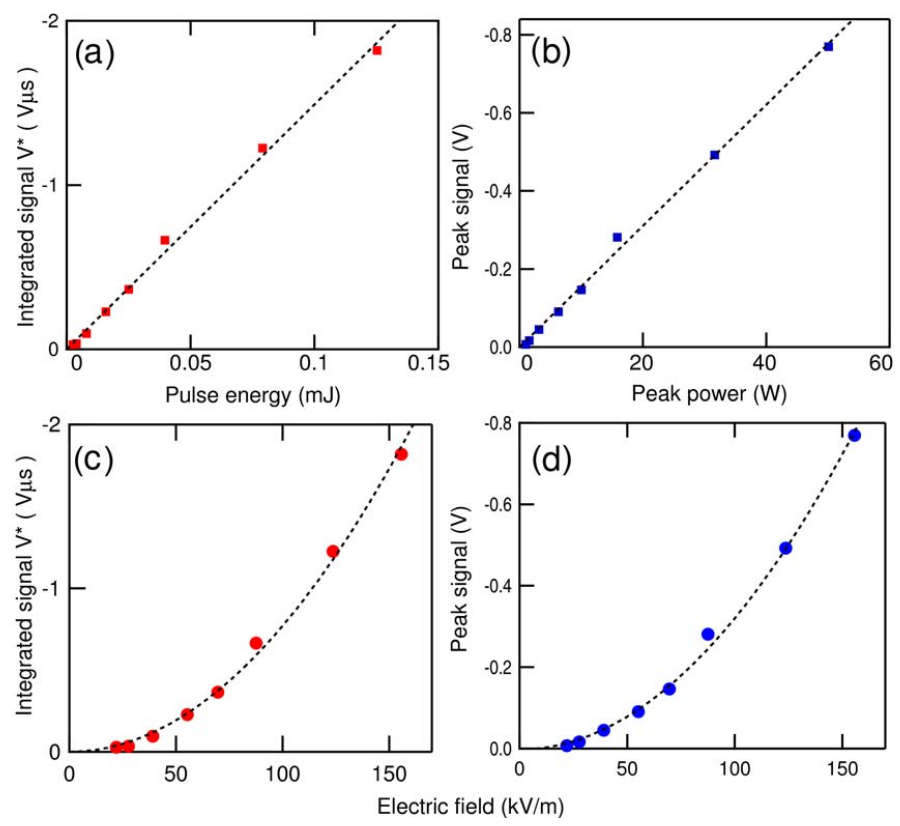

FIG. 6 Integrated signal intensity $V^{*}$ measured with $V_{g 0}=-3.8 \mathrm{~V}$ as a function of the pulse energy and peak signal as a function of the peak power, both of them reduced by a $40 \mathrm{~dB}$ variable attenuator. The data fit with a square-law electric field amplitude detection. An estimate of the responsivity can be derived by the bottom plots as a function of the electric field value obtained by a simple model (see text).

ing wires are at $z<0.5 \mathrm{~mm} \sim \lambda / 4$, well inside the nearfield region of the open waveguide end. We can therefore approximate the peak value of the modulus of the E-field at the transistor position with its value inside the waveguide. The peak value of $\mathbf{E}$ in the waveguide is $220 \mathrm{kV} / \mathrm{m}$, resulting in an output peak voltage of about $3 \mathrm{~V}$. If we consider an effective device length of $1 \mathrm{~mm}$ (including the pick-up wire), we obtain a conversion gain in the range of $10^{-2}$, a factor of ten lower than that of commercial Schottky diode video detectors that we use as monitors. This restricts the use of our device to high-power applications at the moment. It is clear that higher mixing signals can be obtained with device designs which increase $\partial g_{m} / \partial v_{g c}$ like a large number of parallel channels in a small detector area (interdigitated schemes) and/or different heterostructure engineering. Futhermore, a better coupling of the radiation to the channel can be obtained by an optimized antenna design, which was beyond the scopes of the present work.

The responsivity and noise equivalent power (NEP) of the present detector can be calculated in the special condition where one single bonding wire of length $\ell \sim \lambda / 2=1 \mathrm{~mm}$ is coupling most of the $0.15 \mathrm{THz}$ signal into the HEMT channel. We consider the case in Figure 4(c) when the open-end WG is on top of the center of the gate wire, giving at $V_{g}=-4.5 \mathrm{~V}$ a peak signal $\left|V_{d}\right|=2 \mathrm{~V}$. The peak power density $p$ of the incident radiation can be taken as approximately constant along the wire and is $p=|\mathbf{E}|^{2} / Z_{0}=128 \mathrm{~W} / \mathrm{mm}^{2}$. The effective area of a half-wave dipole antenna is given by the classical formula $A_{\text {eff }}=(\lambda / 2)(0.26 \lambda)=0.52 \mathrm{~mm}^{2}$, so that the actual power coupled to the HEMT channel is $P=p A_{\text {eff }}=67 \mathrm{~W}$ and the effective voltage responsivity $R_{v, e f f}=\left|V_{d}\right| / P=0.03 \mathrm{~V} / \mathrm{W}$. Note that this value can be very far from the ideal responsivity, which is obtained when the antenna impedance matches 
the channel impedance at $0.15 \mathrm{THz}$. No effort was made in the present work in order to optimize the responsivity of the detector. In order to calculate the NEP, we assume that the intrinsic equivalent input noise of our HEMT $e_{n}=1.34 \mathrm{nV} / \mathrm{Hz}^{0.5}$ is further amplified by the transistor circuit to a voltage noise at the drain of $V_{n}=e_{n} \times g_{m} \times R_{d}=27 \mathrm{nV} / \mathrm{Hz}^{0.5}$. The calculated $\mathrm{NEP}$ is then given by $V_{n} / R_{v, \text { eff }}=0.9 \mu \mathrm{W} / \mathrm{Hz}^{0.5}$. As one can see, this value is low enough to perform the imaging experiments presented in this work, where we optimized the output swing of our device in the range of $0.1-10 \mathrm{~V}$. We would like to point out that the NEP could be made much lower by increasing the effective responsivity with an optimized antenna design and a proper impedance matching, an issue that we did not consider in our experiment.

The use of transistors as radiation detectors in the microwave range is well known [19] and usually relies on the superheterodyne mixing of a weak radiofrequency (RF) signal with a more intense local oscillator drive (LO), capable of modulating the transistor parameters, in order to obtain a downconverted signal whose intensity is proportional to that of the RF signal. Using high electron mobility transistors (HEMTs), detection of weak RF signals up to the Q-band (40-50 GHz) was reported [18]. In constrast to this use, in the present work the propagating radiation is free-space coupled to the transistor, with no use of high frequency ports and connectors, which allows the detection of radiation by the same HEMT at any frequency which is supported by the intrinsic detection mechanism. Indeed, the free-space coupling principle is also exploited in high-sensitivity mixers developed for space and astronomy applications in the 0.1-6 THz range with $\mathrm{LO}$ and RF signals optically coupled to the mixer [20]. These devices include superconducting bolometers [22]-[24], Josephson tunnelling junctions [25], GaAs Schottky diodes [26]. However, none of these technologies would allow direct integration of electronics for readout and/or room temperature operation. Numerous experiments [1]-[4] suggest that direct detection based on self-mixing may work in HEMTs up to frequencies as high as $10 f_{T}$ with different responsivity values according to the material, the gate length, the antenna coupling, making HEMT focal plane arrays a very promising technology for video-rate imaging in the terahertz range with much room for optimization.

\section{CONCLUSIONS}

In conclusion, we reported the use of a AlGaN/GaN high electron mobility transistor as as a direct radiation power detector at $0.15 \mathrm{THz}$, well beyond its unity gain frequency of $30 \mathrm{GHz}$. We proposed a detection mechanism based on self-mixing due to the high-frequency transconductance change which takes place when antenna-like structures couple a $0.15 \mathrm{THz}$ current into the active channel. We have also shown that the performance of this class of devices can be dramatically improved by using devices with higher transconductance variation with the gate bias and by optimizing the antenna coupling, which we studied here by our near-field active imaging setup. Our work contributes to the development of a new class of transistor-based integrable detectors of radiation up to the terahertz range.

\section{References}

[1] A. Lisauskas, W. von Spiegel, S. Boubanga-Tombet, A. El Fatimy, D. Coquillat, F. Teppe, N. Dyakonova, W. Knap, and H. G. Roskos, "Terahertz imaging with GaAs field-effect transistors" Electron. Lett. 44, 408 (2008).

[2] W. Knap, V. Kachorovskii, Y. Deng, S. Rumyantsev, J.-Q. Lü, R. Gaska, M. S. Shur, C. Simin, X. Hu, M. Asif Khan, C. A. Saylor, and L. C. Brunel, "Nonresonant detection of terahertz radiation in field effect transistors" J. Appl. Phys. 91, 9346 (2002).

[3] F. Teppe, W. Knap, D. Veksler, M. S. Shur, A. P. Dmitriev, V. Yu. Kachorovskii, and S. Rumyantsev, "Room-temperature plasma waves resonant detection of sub-terahertz radiation by nanometer fieldeffect transistor" Appl. Phys. Lett. 87052107 (2005).

[4] A. El Fatimy, F. Teppe, N. Dyakonova, W. Knap, D. Seliuta, C. Valusis, A. Shchepetov, Y. Roelens, S. Bollaert, A. Cappy, and S. Rumyantsev, "Resonant and voltage-tunable terahertz detection in InGaAs/InP nanometer transistors" Appl. Phys. Lett. 89, 131926 (2006)

[5] M. Lee, M. C. Wanke, and J. L. Reno, "Millimeter wave mixing using plasmon and bolometric response in a double-quantum-well fieldeffect transistor" Appl. Phys. Lett. 86, 033501 (2005).

[6] M. Peroni, P. Romanini, A. Pantellini, L. Mariucci, A. Minotti, G. Chione, V. Camarchia, E. Limiti, A. Serino, and A. Chini, "Design, Fabrication and Characterization of Gamma-gate GaN HEMT for High-Frequency/Wide-Band applications" Proceedings of 31st Workshop on Compound Semiconductor Devices and Integrated Circuits (WOCSDICE), 371-378 (Venice, Italy, 2007).

[7] F. Ciocci, R. Bartolini, A. Doria, G. P. Gallerano, E. Giovenale, M. F. Kimmitt, G. Messina, and A. Renieri, "Operation of a compact freeelectron laser in the millimeter-wave region with a bunched electron beam" Phys. Rev. Lett. 70, 928 (1993).

[8] A. Doria, G. P. Gallerano, M. Germini, E. Giovenale, A. Lai, G. Messina, I. Spassovsky, F. Valente, and L. D’Aquino, “Reflective terahertz imaging at the ENEA FEL facility" IEEE P. IRMMW-Thz. 255 (2005).

[9] A. Coppa, V. Foglietti, E. Giovine, A. Doria, G.P. Gallerano, E. Giovenale, A. Cetronio, C. Lanzieri, M. Peroni, and F. Evangelisti, "Active electric near field imaging of electronic devices" Infrared Phys. Techn. 51, 470 (2008).

[10] J. Dyson, "Measurement of near fields of antennas and scatterers" IEEE T. Antenn. Propag. 21, 446 (1973).

[11] A. D. Yaghijan, "Efficient computation of antenna coupling and fields within the near-field region" IEEE T. Antenn. Propag. 30, 113 (1982).

[12] M. Sakowicz, J. Lusakowski, K. Karpierz, M. Grynberg, W. Knap, and W. Gwarek, "Polarization sensitive detection of $100 \mathrm{GHz}$ radiation by high mobility field-effect transistors" J. Appl. Phys. 104, 024519 (2008).

[13] A. Boag, Y. Shimony, and R. Mittra, "Dual band cavity-backed quarter-wave patch antenna" IEE P-Microw Anten P, 2124 (1995).

[14] T. D. Ormiston, P. Gardner, and P. S. Hall, “Microstrip short-circuit patch design equations" Microw. Opt. Techn. Let. 16, 12 (1997).

[15] K. S. Yngvesson, "Ultrafast two-dimensional electron gas detector and mixer for terahertz radiation" Appl. Phys. Lett. 76, 777 (2000).

[16] J.-Q. Lü and M. S. Shur, "Terahertz detection by high-electronmobility transistor: Enhancement by drain bias" Appl. Phys. Lett. 78, 2587 (2001).

[17] R. A. Pucel, D. Masse, and R. Bera, "Performance of GaAs MESFET Mixers at X Band" IEEE T. Microw. Theory 24, 351 (1976). 
[18] M. Kim, J. B. Hacker, E. A. Sovero, D. S. Deakin, and J. H. Hong, “A millimeter-wave multifunction HEMT mixer" IEEE Microw. Guided W. 9, 154 (1999)

[19] J. Zawels, "The transistor as a mixer" P. IRE 42, 542 (1954).

[20] T. G. Phillips and D. P. Woody, "Millimeter- and submillimeterwave receivers" Annu. Rev. Astron. Astr. 20, 285 (1982).

[21] H. R. Fetterman, P. E. Tannenwald, B. J. Clifton, C. D. Parker, W. D. Fitzgerald, and N. R. Erickson, "Far-IR heterodyne radiometric measurements with quasioptical Schottky diode mixers" Appl. Phys. Lett. 33, 151 (1978).

[22] G. N. Goltsman and D. N. Loudkov, "Terahertz superconducting hot-electron bolometer mixers and their application in radio astronomy" Radio. Quantum. Electron. 46, 604 (2004).

[23] A. D. Semenov, H.-W. Hübers, J. Schubert, G. N. Goltsman, A. I.
Elantiev, B. M. Voronov, and E. M. Gershenzon, "Design and performance of the lattice-cooled hot-electron terahertz mixer" J. Appl. Phys. 88, 6758 (2000).

[24] P. Khosropanah, J. R. Gao, W. M. Laauwen, M. Hajenius, and T. M. Klapwijk, "Low noise NbN hot electron bolometer mixer at 4.3 THz" Appl. Phys. Lett. 91, 221111 (2007).

[25] J. Kawamura, J. Chen, D. Miller, J. Kooi, J. Zmuidzinas, B. Bumble, H. G. LeDuc, and J. A. Stern, "Low-noise submillimeter-wave NbTiN superconducting tunnel junction mixers" Appl. Phys. Lett. 75, 4013 (1999).

[26] P. H. Siegel, R. P. Smith, M. C. Graidis, and S. C. Martin, “2.5-THz GaAs monolithic membrane-diode mixer" IEEE T. Microw. Theory 47, 596 (1999). 